\title{
Industry 4.0. Transformation Challenge in Light of Dynamic Capabilities
}

\author{
Kalevi Kyläheiko and Päivi Maijanen
}

\section{INTRODUCTION}

The manufacturing industry is changing drastically due to the changes brought about by the driving forces of the so-called Industry 4.0 Transformation, such as digitalization, modularization, additive manufacturing (3D printing), robotics, artificial intelligence, mass customization, global keen rivalry, etc. Perales et al. [1] characterize Industry 4.0 . by means of virtualization, interoperability, automation, flexibility, real-time availability, service orientation, and energy efficiency, whereas Zezulka et al. [2] emphasize (1) digitization and integration of

K. Kyläheiko $(\bowtie)$

University of Bremen, Bremen, Germany

LUT University, Lappeenranta, Finland

e-mail: kalevi.kylaheiko@lut.fi

P. Maijanen

LUT University, Lappeenranta, Finland

e-mail: paivi.maijanen-kylaheiko@lut.fi

(C) The Author(s) 2020

M. Collan, K.-E. Michelsen (eds.), Technical, Economic and Societal

Effects of Manufacturing 4.0,

https://doi.org/10.1007/978-3-030-46103-4_9 
networks, (2) digitization of product and services, and (3) generating new market (preferably business) models. This means that the firms that want to sustain their competitive advantage (CA) have to be able to rapidly reorganize and transform their resource (especially knowledge) bases and operational manufacturing skills, routines, and capabilities in a cost efficient way. Large multinational companies as well as more local small and medium-sized enterprises in the manufacturing industry have to proactively find a way to re-invent their new business models in a way that combines their strengths in product design and manufacturing together with existing, or to-be-created new capabilities in managing digital ecosystems. Because of strong network externalities (resulting in "the winner takes it all"-earning logic) one can anticipate that only the companies that are the first ones to reinvent, recombine and finally standardize new capability combinations as necessary bottleneck complementary assets will be able to sustain their CA.

Teece [3] uses "digital convergence" as an umbrella concept that touches upon the main aspects of the digital revolution. In its core is wireless communication based on digital broadband technologies. It makes it possible to effectively and flexibly control and monitor extensive platforms and digital ecosystems. Digital information is not locally constrained anymore, which means that the firms can flexibly locate their manufacturing activities so that they can best satisfy individual needs of buyers/customers. The sensors, microprocessors, learning algorithms, etc. enable the firms to remote control complex supply chain networks and manufacturing design problems as well as to anticipate of potential problems already before anything fatal takes place (the rapid rise of the so called "Internet of Things, IoT" manifests that). This means that large parts of manufacturing products can be designed where the best high-tech expert knowledge exists and then flexibly produced where the demand is, for instance, by means of additive manufacturing (3D printers) and robots. Because the role of manual unskilled workers becomes less important, manufacturing becomes more foot-loose thus allowing the rise of "born globals" even amongst small and middle-sized enterprises. Because of digitalization of production design, also the border line between products and services partly loses its importance. The manufacturing firms are also service providers-the integration of manufacturing and service is called servitization in [4] and they show empirically that there seems to be a nonlinear $\mathrm{U}$-shaped interaction-effect between digitalization and servitization on financial performance in a sample of 131 manufacturing companies. In addition, the industry boundaries are losing their distinctive nature and, in 
fact, many of the most promising opportunities can be found on the interfaces between former industry clusters.

Information technologies, banking and finance as well as retailing have been the forerunners in digital convergence. They have managed to put together internet, wireless communication and all kinds of wireless services from music, movies, and cameras to social media (see [3]). The same concept, internet + retailing (Amazon, Alibaba, and eBay), internet + hoteling $(\mathrm{AirBnB})$, internet + taxi (Uber) seems to be working also in other services. They all are also good examples of how modern platforms and digital ecosystems utilize positive network externalities and how the "winner takes it all logic" works.

The manufacturing industry has been much slower at exploiting the huge opportunities of digitalization and wireless communication but it is clear that we will quite soon see also there the rise of digital information based manufacturing ecosystems i.e. the rise of Internet 4.0. However, it is not at all clear who will be the winners and losers within these new ecosystems. The sad histories of Kodak and Polaroid tell the story of how an industry leader can rapidly lose its position when facing the challenge of digitalization, if the management is myopic and unable to respond the new challenges $[3,5,6]$. It may happen that the new leaders will come from outside. For instance, some recent endeavors of Google clearly show that they are eager to take steps to this direction.

Even if we will not go deeper into social issues of Industry 4.0 in this section, it is worth noting one important implication of Industry 4.0.Transformation. The extensive adoption of advanced manufacturing methods will necessarily result in large re-allocations of global labor force working nowadays in manufacturing companies. First, extensive robotics, great flexibility and remote wireless control of digitally designed products/services mean that the role of unskilled labor will be diminishing at the same time, as the role of skilled labor and high-tech experts will be increased. Second, we will also see large geographical relocations, since the importance of labor costs, i.e. wage differentials between countries will not be so important determinants of the location decision as they used to be. For instance, if in an emerging country, let's say India, the labor cost is $50 \%$ lower than the labor cost of Germany and the share of labor cost is $40 \%$ of the value of the product, then the cost advantage of manufacturing the product in India is $20 \%$. This cost advantage most likely covers all the extra re-location costs (transaction costs included) and results in outsourcing manufacturing activities to India. However, if the launching 
of robots etc. reduces the share of manufacturing labor costs from $40 \%$ to $10 \%$, then the cost advantage is only $5 \%$ that hardly covers all extra cost of outsourcing. In this situation, there are no incentives anymore to outsource and we will likely see global supply networks becoming more regional again, more about this, see [7]. This again means that it is most likely that outsourcing is not playing a great role during the era of Industry 4.0. Much more important is the location of high-tech science-based knowledge. This will make advanced manufacturing companies foot-loose and the so-called high wage rate countries will be winners.

Because of the new phenomena and mechanisms that digital convergence creates for the manufacturing firms, it is of great importance to try to understand how they could sustain their CA also in the digital era. In order to give answers to this fundamental question, we will look at the tools that modern strategic management can offer to successfully overcome the transformation challenge. The main challenge that the companies are facing is the challenge of organizational renewal under the circumstances of radical uncertainty. After carefully analyzing the pros and cons of different approaches, we will conclude that the dynamic capability view (DCV) launched by Teece and others [8] is the most suitable approach to analyze the challenge. When dealing with the Industry 4.0 Transformation from the managerial and organizational perspective one can conclude that it is mainly about how to create dynamic capabilities that are able to change path-dependent operational manufacturing capabilities and resource bases in a way that enables a company to sustain its CA [8-11].

This article is organized as follows. First, we will briefly analyze the different approaches of strategic management and look at their general managerial implications. Then we will focus on DCV. Especially its micro foundations will be stressed. In addition, the importance of the Schumpeterian [12] entrepreneurial attitude, i.e., the ability to create "new combinations" as an important precondition to overcome transformation challenges will be discussed. The next section goes further and deeper and utilizes the Teecean sensing-seizing-reconfiguring framework in the context of the digital ecosystems. The main question here is how to profit from innovation in networked ecosystems faced by the firms of the Industry 4.0. We will look at the ways how the firms can create and capture value in these conditions where new kinds of dynamic capabilities and new business models are needed. Finally, some important managerial 
implications and conclusions concerning the ways to overcome the transformation challenge of Industry 4.0. will be offered.

\section{On Different Strategic Management Approaches When FaCing THE Industry 4.0. Transformation Challenge}

All the economics-based strategic management approaches attempt to answer the fundamental question of how to achieve and sustain CA i.e. why some firms are able to outperform others. Basically, there are three explanations for sustainable extra profits (or rents):

(a) Monopoly-based rents are based on product or service market imperfections and the main strategic message is to position a firm so that it maximizes its monopoly (bargaining) power at the same time as it minimizes the monopoly efforts of rival companies. Porter [13, 14] brought these ideas into strategy research by means of his famous "five forces model".

(b) Scarcity-based rents in turn are based on factor or resource market imperfections (instead of Porterian product market imperfections). Following the old ideas of David Ricardo [15], the resource-based view $(\mathrm{RBV})$ posed this issue in the mid 1980s in strategy research [16-18]. Barney [19] summarized the basic managerial message as follows: try to base your competitive advantage on the resources with $\mathrm{V}$ (valuable), $\mathrm{R}$ (rare), I(inimitable), and $\mathrm{N}$ (non-substitutable) attributes. In other words, a firm is able to sustain CA, if it employs resources that create value (meaning that someone is willing to pay for their services) and are scarce and hard to imitate and substitute.

(c) Entrepreneurial rents are based on the firm's ability to find Schumpeterian [12] new combinations i.e. to utilize its resource and knowledge bases in a new way that create new earning opportunities. The dynamic capability view (DCV) introduced by Teece and others [8] opened up this evolutionarily inspired way of thinking in modern strategy research. The most important difference when compared to the Porterian or resource-based view is the dynamic nature of this approach. 
Next, we will briefly discuss the pros and cons of three different CA explanations in the context of Industry 4.0. Transformation.

(a) The Porterian five forces model is based on the microeconomicsbased monopoly model and tries to maximize the bargaining power of the firm. Porter $[13,14]$ introduces three strategies to obtain CA: (1) cost advantage strategy based on economies of scale and scope, (2) differentiation strategy based on the ability to create brands with inelastic demand, and finally (3) niche-based strategies suitable mainly for small and middle-sized enterprises. When analyzing these strategies in the context of the Industry 4.0. Transformation we are tempted to argue that traditional production-related economies of scale are not of great importance any more, since they are more suitable for the industry model where decreasing average total costs could be achieved by large conglomerates. However, also Industry 4.0. offers economies of scale-based advantages mainly for two reasons that in fact explain the rise of platform-based ecosystems during the last 10-15 years. First, digital products/services are often characterized by high first copy cost and then rapidly decreasing marginal costs often approaching zero. This combination creates strictly decreasing average costs and, consequently, a decreasing supply curve. Second, digital goods are also characterized by strong demand-related positive network externalities resulting in the increasing demand curve (up to a certain point). Together these two elements often lead to the "winning takes it all" equilibrium where one company or few oligopolies dominate global markets (think about Google, Facebook, Amazon, Alibaba, Airbnb, etc.). Hence, the main lesson for the companies facing the Industry 4.0. Transformation is to try to simultaneously utilize both the decreasing average costs and (up to a certain point) increasing demand curve.

In addition, the economies of scope are of importance in the era of Industry 4.0, too. If a company is very good at doing something special because of its strong core capabilities, they should try to find other industries (or in fact platforms/ecosystems) in which they can apply them as well. Flexibility, digitalization and globalization of the new industrial world create many new opportunities to exploit this potential. Differentiation strategy can also be utilized during the Industry 4.0. era, since the digitalization/mass 
customization/servitization all create more opportunities to be global instead of being regional as it used to be in the older manufacturing model. The same holds true for the niche-creation strategy. There are opportunities for agile "born globals" as well.

(b) Next, we will have a look at the interpretations offered by the $\mathrm{RBV}$. As mentioned, it is based on the land rent ideas of Ricardo from the year 1817. Instead of focusing on product market imperfections RBV focuses on factor market imperfections. If a company manages to have resources with VRIN (valuable, rare, inimitable, non-substitutable) attributes, it is able to have at least temporary CA [19]. The stronger the so-called isolation mechanisms based, for instance, on causal ambiguity or tacit knowledge [16] are, the better the company is able to establish sustainable CA. Unfortunately, it seems that during the era of Industry 4.0., the opportunities to base CA solely on tangible VRIN resources seem to be very limited. However, the opportunities to utilize knowledge-based intangible assets as elements of CA are much higher. This advantage often utilizes strong and effective software algorithms in order to create totally new customer-tailored services with strong positive network externalities discussed before. In our view, the main message in the context of the Industry 4.0. Transformation has to be reanalyzed and rewritten but, clearly, the intangible resources with VRIN attributes still remain as important sources for CA.

(c) Finally, entrepreneurial Schumpeterian rents that can be obtained and sustained by means of dynamic capabilities to renew, rethink, create and destroy existing resource and knowledge bases to better respond to the challenges of rapid environmental changes are of great importance when trying to face the challenges of the Industry 4.0. Transformation.

After this introduction to the basic ideas of modern strategic management that show that they all are relevant when trying to utilize strategic options created by the Industry 4.0. Transformation, we will now go further by concentrating mainly on dynamic capabilities as main sources of entrepreneurial Schumpeterian rents. Nevertheless, we have to keep in mind that the strategic elements revealed by the Porterian and RBV have to also be taken into account. 


\section{Dynamic Capabilities: What Are They All About}

When analyzing the importance of DCV it is advisable to start with a broader picture that sheds light on its evolutionary roots. In their influential book, "Evolutionary Theory of Economic Growth" Nelson and Winter [20] launched the idea about the firms that consist of routines and more collective bundles of routines, called capabilities. They are stable learned patters that enable a company to be successful. Because of bounded rationality [21] and often even radical uncertainty, the firms are not normally able to optimize. Instead, they try to find satisficing solutions (based on earlier success) that can be improved by continuous learning.

There are different kinds of routines and capabilities. The simplest ones, the so-called first-order capabilities, are generated for pure replication of the existing system $[20,22]$. If the environment remains stationary more than lower-order capabilities are not needed. Of course, however, normally the firms are living in continuously changing environments, which means that replication is not enough to be profitable. The firms have to generate also higher-order capabilities that are able to renew and change existing resource and knowledge bases. These higher-order capabilities are called dynamic capabilities. In fact, we would like to categorize capabilities as a continuum in which they range from pure replicating capabilities via semi-dynamic capabilities ("best practices") to genuine or radical dynamic capabilities that are able to generate new innovative ways to organize business activities.

In order to offer an even broader evolutionary picture we will briefly utilize the cultural evolutionary framework introduced by Campbell [23]. He distinguishes three evolutionary mechanisms that control cultural evolutionary processes, to which also business evolution belongs. The three basic mechanisms are: variation, retention and selection. In the business ecosystem, the role of variation is based on the firms' ability to generate something new or, as Schumpeter [12] put it, to create "new combinations" or innovations. Here the role of entrepreneurial attitude is of great importance. The second mechanism is retention or replication, which creates stability within the firm. Retention is typically realized by lower-order capabilities based on cumulative learning and repetitive actions following the idea of Simonian [21] "satisficing". In a way, one can think that retention is based on organizational culture. The third mechanism, selection, takes place through competition so that the fittest capabilities within the company and, finally, the fittest products/services, i.e., the ones that 
customers are willing to pay for are selected through market forces. This is the basic idea of Schumpeterian creative destruction.

In a similar way as in biological evolution in which variation is realized through mutations and retention through inheritance, it is very important that variation and retention are balanced also in the business context. To give an example, if there are too many radical innovations within a company it is most likely that an organization cannot survive because the existing organizational culture cannot cope with too many radical changes. In the biological sphere, the clear analogy is cancer as a result of two radical mutations. Interestingly, modern strategic management literature deals with this balancing problem by means of the concept of ambidexterity. Based on the ideas of March [24] who analyzed the roles of exploitation (based on existing capabilities, i.e., on lower-order capabilities) and exploration (based on new, not-yet-existing capabilities, i.e., on dynamic capabilities), the ambidexterity literature (see [25]) also deals with balancing these two mechanisms in a way that creates success. The more rapidly the business environment is changing the harder it is for management to balance these two tendencies, variation and retention/replication. If you invest too much in exploitation at the expense of exploration, you are not able to adjust to drastic changes in the business environment and, vice versa, if you invest too much in exploration your organization is perhaps not able to follow rapid changes due to path-dependent rigidities/organizational inertia. Clearly, also the firms in manufacturing are now facing the ambidexterity problem.

After a short evolutionary journey, we will now go deeper to look at the nature of dynamic capabilities. Dynamic capabilities were defined as follows by Teece and others [8] “a dynamic capability is the firm's ability to integrate, build, and reconfigure internal and external competences to address rapidly changing environments".

Another well-known definition is given by Eisenhardt and Martin [26] who stress more the "best practice" nature of dynamic capabilities thus in a way describing what we earlier called as semi-dynamic capabilities. They define dynamic capabilities as "the firm's processes that use resourcesspecifically the processes to integrate, reconfigure, gain and release resources - to match and even create market change. Dynamic capabilities thus are the organizational and strategic routines by which firms achieve new resource configurations as markets emerge, collide, split, evolve, and die". Perhaps the most exact definition is the one of Helfat and others [27], "A dynamic capability is the capacity of an organization to 
purposefully create, extend, or modify its resource base". In our view, this definition most clearly emphasizes Schumpeterian entrepreneurial thinking in which "new combinations" i.e. innovations are the engines of strategic (see $[28,29])$.

Teece [9] went further in order to reveal the micro foundations of dynamic capabilities. According to Teece [9-11], dynamic capabilities consist of three separate capacities i.e. sensing, seizing, and reconfiguring (transformation). To generate real changes based on dynamic capabilities the firms have to be able to, first, sense even weak signals that appear as strategic options. Second, they have to be able to invest i.e. to exercise the most promising strategic options. Often this also means that the firms have to disinvest in some older capabilities that are not regarded as profitable any more. Third, the managers have to be able to reconfigure or transform their existent path-dependent resource and knowledge bases and processes in a way that makes it possible to realize strategic options sensed.

Again, it may happen that different capacities of dynamic capabilities are not balanced. For instance, some firms may be very effective in sensing new strategic options by means of entrepreneurial alertness and/or efficient technology scanning systems but at the same time, the managers can be quite too slow to make investment decisions thus destroying the existing opportunities. Perhaps the most problematic part is the third one i.e. how to effectively reconfigure (transform) your existing resource and knowledge bases. It is not enough to do the things right by means of operational and semi-dynamic capabilities but the managers have to do the right things through dynamic capabilities as well. Here we see the ambidexterity tradeoff problem in action. As mentioned, it is of great importance for a company to have a stable organizational culture that is based on continuous learning and exploitation of existing routines and capabilities. On the other hand, transformation necessarily means also explorative actions that destroy at least partly existing path dependent capabilities. This necessarily creates tensions on different organizational levels. There is always a tradeoff between competence-enhancing exploitation and competence-destroying exploration [25]. 


\section{Dynamic Capabilities in Digital Platform-Based Ecosystems: How to Create and Capture Value}

In the previous section, we introduced the evolutionarily inspired view of the way how firms behave and how they can achieve and sustain their CA by means of lower- and higher-level capabilities. However, during the last ten years the things have become even more complicated because of rapid globalization, keener rivalry, product and service mass customization, need of increased flexibility, modularization, digitalization and related positive network externalities. These "digital convergence" [3] related phenomena have dramatically changed the way how the firms nowadays compete with each other and especially how they get connected with each other through the so-called multi-sided platforms (MSP) [30]. The reason of the rapid rise of MSP's relates to positive network externalities resulting in increasing demand curves together with often dramatically decreasing average total costs due to large "first copy cost". The first mover's advantage or "winner takes it all" are the dominating principles of the MPS's. The firms that rapidly achieve the so-called critical mass are also able to establish and dominate their own platforms and thus indirectly create their own ecosystem. Because of network externalities and increased flexibility (mainly due to digitalization), the companies are able to leave their traditional industry clusters and to create quite new interfaces and ecosystems.

There are many definitions for platforms and ecosystems. We will use the ones applied in modern strategic management literature. Teece [3] defines the platforms and ecosystems as follows, "A platform is any combination of hardware and software that provides standards, interfaces, and rules that enable and allow providers of complementors to add value and interact with each other and/or users. Collectively, the platform innovator(s) and the complementors constitute an ecosystem, the viability of which depends on continued innovation and maintenance of the platform by its owner(s) and a delicate balance of cooperation and competition among the providers of complements". Helfat and Raubitschek [30] stress that "digital MSP ecosystems are characterized by crosside (or indirect) network effects, in which the value to a party on one side of the platform depends on the number and quality of the parties on the other side(s) of the platform. Cross-side network effects are often positive". Complementary assets play an important role in each MSP's and they are essential when a firm tries to build its business model in order to profit from its innovation. A succinct definition of the concept ecosystem is 
offered by Adner [31]. He regards it as "an alignment structure of the multilateral set of partners that need to interact in order for a focal value proposition to materialize". Alignment structures may be variable and the structure is often non-hierarchical consisting of complementarities that are co-specific and multi-sided. It is worth noting that partners in an ecosystem can simultaneously be also rivals.

Because of rapid digitalization, platforms and related ecosystems are becoming pervasive and therefore a provider of a complementary asset(s) has to be able to become a part of a wider platform. Even if the platforms often are multi-sided, it is typical that there is a technologically leading company that sets the agenda and controls the evolution of the platform. As Teece [32] states, an ecosystem is anchored by a platform or many rival platforms that are connected through common standards (often protected by patents, copyrights, trade secrets, etc.) and interfaces.

It is interesting to note that the idea of an ecosystems fits very well to the evolutionary theory of the firm launched in the former section. The basic idea is based on biological co-evolution, a process through which species become developed in a continuous evolutionary cycles following the variation, selection, and retention mechanisms. Moore [33] first adopted this idea in business literature in a Harvard Business Review article. Of course, Nelson and Winter [20] utilized the same idea in their evolutionary models, even if they did not use the concept of an ecosystem. As Teece [32] highlights, co-creation and co-evolution through competition (selection) are typical also for business ecosystems in which the innovator has to make transaction cost based decisions (see also [34]) about the elements of the value creating platform. The main question is which innovative ideas are to be internalized and which ones to be externalized for other providers of complementary assets.

Focusing on the importance of complementarities Jacobides and others [35] state succinctly "An ecosystem is a set of actors with varying degrees of multilateral, non-generic complementarities that are not fully hierarchically controlled". Especially they stress that the relevant complementaries can be co-specialized and unique (i.e. the items $\mathrm{A}$ and $\mathrm{B}$ cannot be produced alone without coordination that puts them together) and/or supermodular (i.e. the more an item $\mathrm{A}$ is produced the cheaper or better in quality are also the items B and C). Uniqueness and supermodularity mean that interdependencies are standardized, which in turn presupposes specific routines and capabilities that are needed in designing ecosystems. Helfat and Raubitschek [30] call them integrative capabilities and 
especially the ability to create them is of great importance when trying to profit from platforms and ecosystems.

In his influential Research Policy article, Teece [36] launched the idea of the profiting from innovation framework in order to analyze how a firm could profit from its innovation. Of course, the situation in the 1980s was not that complex as it is nowadays. Teece focused on one product innovation-one company-one industry-model and showed that the most important factors when trying to capture the fruits of innovation were (1) the nature of the appropriability regime (based either on tacit knowledge or on legal means, such as patents, copyrights, trademarks, and trade secrets), (2) the role of co-specialized complementary assets, (3) the nature of innovation (autonomous or systemic), and (4) timing. Appropriability regimes are strong when knowledge assets are based on tacit knowledge and, in addition, protected by legal means. Even in the 1980s, it was clear that legal means were strong only in some industries, such as pharmaceutical or chemical ones. Hence, even then the complementarities and the nature of innovation played a crucial role. Interestingly, Teece [36] in a way came to the same conclusion as the RBV at the same time. If a firm manages to have strongly protected assets that preferably are also bottlenecks in a supply chain, it is most likely able to profit from innovation as well.

In his follow-up article, Teece [3] updated his profiting from innovation framework for the digital era emphasizing the role of network externalities and digital convergence and launching the idea of an ecosystem in which the role of complementarities and multi-inventions are crucial. Teece [3] started now from platforms and ecosystems stressing the fundamental roles of complementarities and positive externalities-based interdependencies that, of course, weakened the role of the traditional appropriability regime based on legal means.

Teece [3] showed that it is hard to protect general-purpose technologies as well as so-called enabling technologies, such as photonics, advanced materials, nanotechnologies, artificial intelligence, machine learning and robotics. This means that there are too little incentives to produce new knowledge in these fields without public funding or without successfully participating in value creating ecosystems. The key factors were the complementarities together with a strong appropriability regime. It is not any more enough that a company is able to innovate in enabling technologies and to protect it strongly by means of tacit knowledge or legal means (cf. [37]). It has to be able to connect it to an existing or emerging digital ecosystem 
and, most importantly, it has to be able to generate a bottleneck asset that is unique and preferably super-modular. As Teece states [3], however, "these bottleneck assets are not easy to identify and they may shift over time" when an ecosystem evolves. A similar situation can be found from many manufacturing platforms, such like the automobiles or aircraft in which modularization and standards have made it hard to profit from autonomous innovation because of keen competition between the providers of complementarities. In fact, in the digital era competition takes place at three levels, first, between the providers of complementary assets within a platform, second, between different platforms within an ecosystem, and third, between rival ecosystems (see also [3]). In addition, all the ecosystems are continuously evolving at the same time when the interfaces are getting more blurred. Interestingly, a company can also be a part of different (even rival) platforms and ecosystems.

As Teece [3] concludes the fate of a company within an ecosystem and the fate of the whole ecosystem now drastically depends on (1) the ability to continuously generate relevant complementarities and especially on (2) the cognitive entrepreneurial capabilities [38] of the ecosystem's leaders to orchestrate, coordinate and strategize the ecosystem. In order to profit from the platforms the firms have to be able to produce bottleneck complementarities that create value for the platform and are somehow protected through tacit knowledge and timing.

This brings us to the issue of dynamic capabilities. Building up competitive platforms or bottleneck complementarities and designing business models in rapidly evolving digital ecosystems is not possible without strong dynamic capabilities. The managers of platform leading companies have to be able to sense new opportunities also outside the platforms, to seize the new opportunities rapidly if needed, and to reconfigure knowledge and resource bases not only within the own company but also within the whole platform or even ecosystem by changing its constituting elements and complements providers. In a similar way, the managers of complementary assets providers have to be able to sense, seize and reconfigure to be able to create critical bottleneck assets.

Helfat and Raubitschek [30] develop these ideas further by analyzing the dynamic capabilities that are necessary for profiting from innovation in digital multi-sided platform-based ecosystems. As they state, the platforms do not automatically generate positive multi-sided externalities but they have to be created through a deliberate design. This is mainly on the responsibility of the leader of the platform. They have to be able to 
orchestrate and coordinate the ecosystem consisting of many at least partly competing complementary asset providers. In addition, they have to continuously develop the "core product/service" of the platform and find the most effective complementary assets providers who are willing to join the ecosystem. This in turn presupposes the ability to balance the different needs of complementors so that they have enough incentives to be innovative. On the other hand, the leading company/companies have to be able to take their own stake. According to Helfat and Raubitschek [30] especially three types of dynamic capabilities are of vital importance for the leaders when trying to cope with multi-sided platform-based ecosystems:

1. Innovative capabilities. Leaders have to be able to develop the core product (product sequencing) but, in addition, they have to be able to integrate new complementors and their knowledge assets in an efficient way.

2. Scanning/sensing capabilities. Of course, the leaders have to sense new opportunities related to the core product(s)/ (service(s) of the ecosystem and to take into account the threats arising from the competitive environment. In addition, they have to be able to scan potential innovation sources that can be created through existing or new potential complementors. On the other hand, the complementary assets providers have to scan and sense new opportunities to make their assets as bottlenecks within the platform.

3. Integrative capabilities relate mainly to designing suitable business models. There are decisions about internalizing knowledge assets between the leader and complementors or between rival complementors i.e. they have to take into account the nature of governance structure based on transaction cost considerations (see $[30,34]$ ). They also have to make decisions on the pricing structures for products/services provided within the ecosystem and between different customers. Integrative capabilities also support interactions and relationships between the members of an ecosystem as well as between ecosystem members and external parties. The more complex and knowledge-intensive interfaces there are and the more rapidly the ecosystem is evolving the more complicated is the task of orchestration. Finally, integrative capabilities are also partly responsible for how effective and innovative the sensing/scanning capabilities are. 
Helfat and Raubitschek [39] launched already earlier the idea of integrative knowledge that is the basis of integrative capabilities as follows: "knowledge that integrates, or knowledge of how to integrate, different activities, capabilities, and products within a vertical chain or across vertical chains."

From the perspective of other members of an ecosystem, the role of innovative and scanning/sensing capabilities are quite similar and, of course, they need integrative capabilities, even if they do not have to take responsibility for the general governance structure of the whole ecosystem. Much more important for them is to focus on creating such a combination of especially innovative and integrative capabilities that enable them to profit from the bottleneck properties of their complementary assets. The better protected, supermodular, and co-specialized their complementary assets are the stronger their bargaining power within the ecosystem is.

\section{Discussions and Managerial Implications}

This article deals with obtaining and sustaining CA in manufacturing firms during the era of digital ecosystems. "Digital convergence" is drastically changing the way how the firms can profit from innovation as the examples from information technology, finance, banking, and retailing clearly manifest. In addition, the rules of the game are dramatically changing. In the future, it will not be any more possible to do everything all alone from the basic innovation to custom-tailored products and services. In the future, also the manufacturing firms have to be able to work together within digital ecosystems that often take the form of multi-sided platforms. On the one hand, these digital ecosystems are often based on positive externalities that make the systems evolve rapidly. This results in high uncertainty and the need for proactive behavior. On the other hand, these platforms and ecosystems are based on standards and very often strict modularization that does not leave very much room for individual actions. This intensifies competition between rival providers of complementary assets and makes the extra profits generated by traditional VRIN resources or autonomous innovations often temporary.

In this article, we have analyzed the nature of digital platforms and ecosystems and the way they function. In addition, we have launched the tools that economics-based strategic management literature can offer in order to achieve and sustain CA and scrutinized how effective they could 
be under the circumstances of digital convergence. Based on this analysis, we will now summarize our main results in the context of Industry 4.0.Transformation. It can be interpreted as a checklist that managers have to take into account when trying to profit from innovation in the digital era.

- The Porterian message: You have to maximize your bargaining power in relation to rivals. This can be based on the economies of scale and scope, differentiation or niche creating strategies. During the digital era most economies of scale are based on demand-related, scalable network externalities. If a company is able to exploit them it may also generate its own platform or even an ecosystem and be the leading partner within it. To be able to do this a firm needs innovative, scanning/sensing and especially integrative capabilities. It also has to be able to continuously evolve its platform and make transaction cost based internalization/externalization decisions. However, if a company is not able to generate scalable network externalities it may perhaps try to use differentiation or niche strategies but preferably as a born global trying to get internationalized as soon as possible by using the tools of Internet 4.0. Transformation. Unfortunately, local advantages based on differentiation or niches cannot be sustainable, even if a temporary CA can perhaps be achieved. This is due to keen competition within the platform and ecosystem.

- Resource-based message: In order to profit from VRIN resources a firm has to be able to find resources that create new value and are hard to imitate. In the manufacturing sector, the autonomous innovations are, however, hard to protect and hence the extra profits (rents) are normally only temporary. But again, if a company has dynamic capabilities (especially innovative, sensing and integrative) it can perhaps generate complementary bottleneck assets that are necessary and supermodular in nature. If a company is lucky, it can really profit from its autonomous innovations, even if there typically exists keen competition between rival providers of complementary assets.

- Capability-based message: First, the managers of manufacturing companies have to understand the evolutionary nature of their company and its role in platforms/ecosystems. It is not any more possible to try to survive all alone. It is necessary to function as a partner in networks and to utilize all the different capabilities from operational via semi-dynamic (best practices) to genuinely dynamic capabilities. 
Most important are the ones that support innovation, scanning of new strategic options, and integration thus enabling to become a partner in platforms/ecosystems.

- The managers should also understand the message of the ambidexterity tradeoff. They have to be able to develop both their operational capability-based exploitation and dynamic capability-based exploration in a balanced way.

- Nevertheless, the more turbulent and rapidly changing the business environment is, the more the firms have to invest in dynamic capabilities. Here the basic logic of the profiting from innovation framework $([3,36,37])$ still holds. In order to gain a CA position a firm has to be able to create ( 1 ) bottleneck complementary assets that are (2) protected either by legal means (nowadays not so important any more) or (3) tacit knowledge embedded into the organizational culture and/or (4) to utilize timing advantage. Bottleneck complementary assets are in the future more and more knowledge-based consisting of operational manufacturing capabilities, integrative and innovative dynamic capabilities as well as science-based knowledge assets. At the same time when regional wage rate cost advantages will lose their importance due to robots, additive manufacturing, etc. the companies become much more flexible and foot-loose. Our guess is that in the future, it is much more important to have close connections to the universities and research centers than try to minimize labor cost differentials. This science-based co-operation presupposes strong integrative dynamic capabilities.

\section{REFERENCES}

1. D. P. Perales, F. A.Valero, and A. B. Garcia, "Industry 4.0, a classification scheme," in Closing the gap between practice and research in industrial engineering, in Closing the gap between practice and research in industrial engineering, edited by E. Viles, M. Ormazabal and A. Lleo, Eds., Cham, Switzerland: Springer, 2018, pp. 343-350.

2. F. Zezulka, P. Marcon, I. Vesely, I., and O. Sajdl, "Industry 4.0 - An Introduction in the phenomenon," IFAC-PapersOnLine, vol. 49, pp. $8-12,2016$.

3. D. Teece, "Profiting from innovation in the digital economy: Enabling technologies, standards, and licensing models in the wireless world," Research Policy, vol. 47, pp. 1367-1387, 2018. 
4. M. Kohtamäki, M., Parida, V., P. C. Patel, P. C., and H. Gebauer, "The relationships between digitalization and servitization: The role of servitization in capturing the financial potential of digitalization”. Technological Forecasting and Social Change. 151, February 2020, 119804.

5. M. Collan, J. Freiling, K. Kyläheiko, and E. Roemer, "Entrepreneurship and the art of tackling technological crises: a strategic real options framework", International Journal of Technology Intelligence and Planning, 10, 166-185.

6. B. Wernerfelt, "A resource-based view of the firm," Strategic Management Journal, vol. 5, pp. 171-180, 1984.

7. J. Rose, "How the U.S. can regain its manufacturing edge? An interview of Justin Rose in Knowledge@Wharton, July 10. 2019," ed, 2019.

8. D. J. Teece, G. Pisano, and A.Shuen, "Dynamic capabilities and strategic management". Strategic Management Journal. 18. 509-533.

9. D. J. Teece, "Explicating dynamic capabilities: the nature and microfoundations of (sustainable) enterprise performance," Strategic Management Journal, vol. 28, pp. 1319-1350, 2007.

10. D. Teece, "The foundations of enterprise performance: Dynamic and ordinary capabilities in an (economic) theory of firms," Academy of Management Perspectives, vol. 28, pp. 328-352, 2014.

11. D. Teece, "Dynamic capabilities: Routines versus entrepreneurial action," Journal of management Studies, vol. 49, pp. 1395-1401, 2012.

12. J. A. Schumpeter, Capitalism, Socialism and Democracy. New York, NY, USA: Harper \& Brother, 1942.

13. M. E. Porter, Competitive Strategy: Techniques for Analyzing Industries and Competitors. New York: The Free Press, 1980.

14. M. E. Porter, Competitive Advantge. New York: The Free Press, 1985.

15. D. Ricardo, The principles of political economy and taxation. London, 1817.

16. R. P. Rumelt, "Towards a Strategic Theory of the Firm," in Competitive strategic management, R. B. Lamb, Ed., ed Englewood Cliffs, NJ, USA: PrenticeHall, 1984, pp. 556-570.

17. J. Barney, "Organizational culture. Can it be a sound source of sustained competitive advantage?," Academy of Management Review, vol. 11, pp. 656-665, 1986.

18. M. Peteraf, "The cornerstones of competitive advantage: A resource-based view," Strategic Management Journal, vol. 14, pp. 179-191, 1993.

19. J. Barney, "Firm Resources and Sustained Competitive Advantage," Journal of Management, vol. 17, pp. 99-120, 1991.

20. R. R. Nelson and S. G. Winter, An evolutionary theory of economic change. Cambridge: Harvard University Press, 1982.

21. H. Simon, "From substantive to procedural rationality," in Method and appraisal in economics, S. Latsis, Ed., ed Cambridge Cambridge University Press, 1976, pp. 129-148. 
22. S. G. Winter, "Understanding dynamic capabilities," Strategic Management Journal, vol. 24, pp. 991-995, 2003.

23. J. Campbell, "Growth Pole theory, diagraph analysis and interindustry relationships," Journal of Economic and Social Geography, vol. 63, pp. 79-87, 1972.

24. J. G. March, "Exploration and exploitation in organizational learning," Organization Science, vol. 2, pp. 71-87, 1991.

25. M. Tushman and P. Andersson, "Technological discontinuities and organizational environments," Administrative Science Quarterly, vol. 31, pp. $439-465,1986$.

26. K. M. Eisenhardt and J. A. Martin, "Dynamic capabilities: What are they?," Strategic Management Journal, vol. 21, pp. 1105-1121, 2000.

27. C. E. Helfat, S. Finkelstein, W. Mitchell, M. A. Peteraf, h. Singh H., D. J. Teece, and S. G. Winter, Dynamic Capabilities: Understanding Strategic Change in Organizations. Malden, MA: Blackwell Publishing, 2007.

28. F. Ardnt and L. Pierce, "The behavioral and evolutionary roots of dynamic capabilities," Industrial and corporate change, vol. 27, pp. 413-424, 2018.

29. O. Schilke, S. Hu, and C. E. Helfat. "Quo vadis, dynamic capabilities? A content-analytic review of the current state of knowledge and recommendations for future research". Academy of Management Annals, 12(1), 390-439.

30. C. Helfat and R. S. Raubitcheck, "Dynamic and integrative capabilities for profiting from innovation in digital platform-based ecosystems," Research Policy, vol. 47, pp. 1391-1399, 2018.

31. R. Adner, "Ecosystem as structure: An actionable construct for strategy," Journal of Management, vol. 43, pp. 39-58, 2017.

32. D. Teece, "Next generation competition: New concepts for understanding how innovation shapes competition and policy in the digital economy," Journal of law, economics, and policy, vol. 9, pp. 97-118, 2012.

33. J. F. Moore, "Predators and prey: a new ecology of competition," Harvard business review, vol. 71, pp. 75-86, 1993.

34. M. G. Jacobides and S. Winter, "The co-evolution of capabilities and transaction costs: Explaining the institutional structure of production," Strategic Management Journal, vol. 26, pp. 395-413, 2005.

35. M. G. Jacobides, C. Cennamo, and A. Gawer. "Toward a theory of ecosystems". Strategic Management Journal. 39, 2255-2277.

36. D. Teece, "Profiting from technological innovation: Implications for integration, collaboration, licensing and public policy," Research Policy, vol. 15, pp. 285-305, 1986.

37. D. Teece, "Reflections on "Profiting from innovations"," Research Policy, vol. 35, pp. 1131-1146, 2006.

38. C. Helfat and J. A. Martin, "Dynamic managerial capabilities: Review and assessment of managerial impact on strategic change," Journal of Management, vol. 41, pp. 1281-1312, 2015.

39. C. Helfat and R. S. Raubitcheck, "Product sequencing: knowledge, capabilities, and products," Strategic Management Journal, vol. 21, pp. 961-979, 2000. 
Open Access This chapter is licensed under the terms of the Creative Commons Attribution 4.0 International License (http://creativecommons.org/licenses/ by $/ 4.0 /$ ), which permits use, sharing, adaptation, distribution and reproduction in any medium or format, as long as you give appropriate credit to the original author(s) and the source, provide a link to the Creative Commons licence and indicate if changes were made.

The images or other third party material in this chapter are included in the chapter's Creative Commons licence, unless indicated otherwise in a credit line to the material. If material is not included in the chapter's Creative Commons licence and your intended use is not permitted by statutory regulation or exceeds the permitted use, you will need to obtain permission directly from the copyright holder.

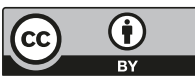

